Bulletin d'Histoire Contemporaine de

l'Espagne

$51 \mid 2017$

Les forces politiques durant la Seconde République espagnole

\title{
Ensayo bibliográfico: dos décadas de historiografía política sobre la Segunda República
}

Juan Avilés Farré

\section{(2) OpenEdition \\ Journals}

Edición electrónica

URL: http://journals.openedition.org/bhce/725

DOI: 10.4000/bhce.725

ISSN: 1968-3723

\section{Editor}

Presses Universitaires de Provence

\section{Edición impresa}

Fecha de publicación: 1 junio 2017

Paginación: 151-161

ISSN: 0987-4135

\section{Referencia electrónica}

Juan Avilés Farré, «Ensayo bibliográfico: dos décadas de historiografía política sobre la Segunda República », Bulletin d'Histoire Contemporaine de l'Espagne [En línea], 51 | 2017, Publicado el 09 octubre 2018, consultado el 20 abril 2019. URL : http://journals.openedition.org/bhce/725 ; DOI : 10.4000/ bhce.725 


\title{
Ensayo bibliográfico: un cuarto de siglo en la historiografía política sobre la Segunda República
}

\author{
Juan AVILÉS FARRÉ
}

UNED

El avance acelerado de la investigación en aquellos campos que despiertan el interés de los historiadores y de su público hace que en un cuarto de siglo pueda renovarse por completo el conocimiento de un período y esto es lo que ha ocurrido en el caso de la Segunda República española. En este breve ensayo trataré de dar cuenta de algunas de las publicaciones más importantes que sobre el tema han aparecido entre 1991 y 2014, sin ánimo de exhaustividad y sin la pretensión de no haber omitido obras que también son relevantes, pero con el propósito de que queden reflejadas las diferentes tendencias historiográficas y los principales campos de interés. A fin de acotar el tema, he prescindido de los artículos publicados en revistas, que a menudo ofrecen la primicia de una investigación, pero cuya información suele ser luego reelaborada por sus autores en forma de libro, ya que sigue siendo éste el principal medio de difusión del conocimiento histórico. Y he prescindido también de los estudios de ámbito local, que representan uno de los campos más importantes en el avance de la investigación, pero cuyo análisis detallado habría exigido un ensayo de dimensiones mucho más amplias.

Mencionaré en primer lugar algunas obras que proporcionan una visión de conjunto del período republicano, bien hasta el estallido de la guerra civil en 1936, bien hasta la caída de la República en 1939. Una aportación temprana, dentro del período considerado, la realizó el historiador estadounidense Stanley Payne, en su libro Spain's first democracy: the Second Republic, 1931-1936 (1993), cuyo capitulo final respondía a la cuestión básica de por qué fracasó la República, para responder a la cual aludía a factores económicos y sociales de tipo estructural, a un factor coyuntural, como fue la coincidencia temporal con un momento de máxima intensidad del conflicto sociopolítico en Europa, y sobre todo a factores estrictamente políticos, es decir a la incapacidad de las distintas fuerzas políticas para atenerse a los principios del consenso democrático. El ponderado, breve y bien documentado libro de Julio Gil Pecharromán, Historia de la Segunda República española, 1931-1936 (2002), recurre menos a los juicios de valor y no ofrece un diagnóstico del fracaso republicano, pero sí una síntesis clara de los orígenes de la República, de la coyuntura económica y social, de la articulación del Estado republicano, de su política exterior y de cada una de las etapas en que se desarrolló su convulsa historia. Con estilo más narrativo y una mayor propensión a interpretar y valorar, el libro de Gabriele Ranzato L'eclissi della democracia: la guerra civile spagnola e le sue origini, 1931-1939 (2004), se plantea también la pregunta de por qué la democracia liberal, que con la República de 1931 había tenido una gran ocasión de afirmarse en España, fracasó de manera tan clamorosa. La primera parte de la respuesta estriba, por supuesto, en la fuerza que adquirieron los enemigos de la democracia, pero Ranzato sostiene que para tener una explicación completa 
de ese fracaso hay que tener en cuenta que sus amigos «carecían de la tradición, la cultura y la madurez necesarias para salvaguardar el sistema democrático, para lo cual habría sido necesario poner limites a los objetivos y aspiraciones populares». Por último, entre las grandes síntesis debidas a un solo autor, es necesario mencionar la de Julián Casanova, República y guerra civil (2007), una obra bien eserita que analiza las dificultades de la República en función del abismo abierto entre mundos culturales antagónicos: «entre católicos practicantes y anticlericales convencidos, amos y trabajadores, Iglesia y Estado, orden y revolución»».

Respecto a las obras colectivas sobre la experiencia republicana en su conjunto, mencionaré en primer lugar las actas de un coloquio internacional celebrado en Roma en 1991, que fueron editadas por Giuliana Di Febo y Claudio Natoli en un volumen, Spagna anni Trenta: società, cultura, istituzioni (1993), que reúne importantes ensayos sobre el debate historiográfico, los movimientos políticos y sociales, las relaciones entre la Iglesia y la sociedad, los intelectuales y la cultura en el período de la República y la guerra civil. Las actas de otro coloquio internacional celebrado diez años después en París, editadas por Marie-Claude Chaput y Thomas Gomez en el volumen Histoire et mémoire de la Seconde République espagnole (2002), abordan también aspectos políticos, económicos y culturales, así como la memoria de la República en la etapa franquista, en el exilio y en la España democrática de hoy. La historia y la memoria de la República, en sus diversas facetas, se analizan así mismo en la obra colectiva coordinada por Ángeles Egido Memoria de la Segunda República: mito y realidad (2006). Por su parte, Santos Julía ha sido el editor de un volumen colectivo que cubre todo el período de los años treinta: República y guerra en España (2006).

Muy recientemente se han publicado varios libros que profundizan en la línea de atribuir el fracaso de la Segunda República a la ausencia de una cultura democrática en la España de los años treinta, es decir en la falta de una voluntad de aceptar que el respeto a los principios democráticos está por encima de los objetivos de cada partido y que los resultados de unas elecciones libres deben ser siempre respetados. En esa línea se inscribe el libro de Manuel Álvarez Tardío y Roberto Villa El precio de la exclusión: la política durante la Segunda República (2010), que destaca como el advenimiento de la República fue vivido por sus protagonistas como un revolución pacífica, es decir como una ruptura con un pasado demonizado, y que el asentamiento de la democracia se resintió de esa convicción, porque la mayoría de los diputados en las Cortes Constituyentes «consideraban virtuosa una actitud contraria a la inclusión de quienes pensaban diferente». Un año después se publicó una obra colectiva dirigida por Fernando del Rey, Palabras como puños: la intransigencia politica en la Segunda República española (2011), cuyo título expresa fielmente su tesis. Incluye una decena de ensayos, entre los que cabe destacar el del propio Fernando del Rey sobre los socialistas, el de Álvarez Tardío sobre la CEDA y el de Pedro González Cuevas sobre el fascismo, mientras que llama la atención la ausencia de un ensayo sobre el azañismo, dado el papel fundamental que Manuel Azaña jugó en la democracia republicana. Tras ello los citados Álvarez Tardío y Rey han unido sus esfuerzos como editores de un nuevo libro colectivo El laberinto republicano: La democracia española y sus enemigos, 1931-1936 (2012), lo que parece marcar la consolidación de una escuela dispuesta a poner en cuestión la interpretación de la Segunda República difundida por la historiografía de izquierdas. Representativas de la posición opuesta son en cambio tres obras colectivas editadas por Manuel Ballarín y otros, que en su conjunto cubren todo el período 1931-1936: Avenida de la República (2007), La II Republica en la encrucijada (2009) y La República del Frente 
Popular (2010). Este último periodo es también abordado desde una perspectiva similar en un libro colectivo coordinado por Eduardo González Calleja y Rocío Navarro, La España del Frente Popular (2012).

Tres aspectos de la historia republicana han atraído especialmente el interés de los investigadores: la cuestión de las autonomías regionales y los nacionalismos, la de las relaciones entre la Iglesia y el Estado y el auge del anticlericalismo, y la de los problemas del orden público y la violencia política, especialmente en los meses que precedieron al estallido de la guerra civil. Sobre el primer tema, que ha adquirido una gran relevancia en la España actual, resulta muy útil la obra colectiva editada por Justo G. Beramendi y Ramón Maíz Los nacionalismos en la España de la II República (1991), que incluye más de veinte ensayos sobre los partidos e ideologías nacionalistas, sus bases sociales y la remodelación del Estado español, que no se limitan a los casos catalán y vasco, sino que se ocupan también de la experiencia, gallega, valenciana, aragonesa y andaluza. Sobre la historia vasca en los años de la República resulta también útil el libro de Juan Pablo Fusi, El País Vasco, 1931-1937: autonomía, revolución, guerra civil (2002), una recopilación de trabajos anteriores del autor que presta especial atención al complejo debate autonómico y la actuación de las organizaciones obreras, mientras que de la magna obra de José Luis de La Granja sobre el nacionalismo vasco me limitaré a destacar aquí la recopilación de ensayos de su libro El oasis vasco: el nacimiento de Euskadi en la República y la Guerra Civil (2007). Acerca del líder del nacionalismo vasco en los años de la República se ha publicado una valiosa biografía Mees, Granja, de Pablo y Rodríguez Ranz, La politica como pasión: el lehendakari José Antonio Aguirre (2014). Una obra interesante sobre un personaje clave del catalanismo republicano es la editada por Teresa Abelló y Josep Maria Solé i Sabaté Lluis Companys, president de Catalunya: biografia humana i politica, Barcelona, 2007, mientras que sobre su partido se ha publicado una extensa obra de Joan Culla i Clarà, Esquerra Republicana de Catalunya (2013), que analiza su evolución hasta nuestros días.

La bibliografía sobre la cuestión religiosa en la Segunda República se ha ampliado de manera considerable en los últimos años. Entre los estudios que examinan la política laicista de la República desde una perspectiva crítica hay que citar Anticlericalismo y libertad de conciencia (2002), un libro en el que Manuel Álvarez Tardío analiza la política religiosa de los gobernantes republicanos y concluye que el anticlericalismo fue «una manifestación más del exclusivismo político», es decir de la negativa de las izquierdas a admitir la pluralidad ideológica de los españoles. Muy diferente es la interpretación de Ángel Luis López Villaverde, quien en El gorro frigio y la mitra frente a frente (2008) se propone «revisar el revisionismo» y plantea un modelo más complejo del conflicto entre la Iglesia y la República, que presta especial atención al nivel local e incluye también los años de la guerra civil. La explicación de la violencia anticlerical ha sido así mismo abordada por José Manuel Barrios en Iconoclastia (2007) y por Maria Thomas en La fe y la furia (2014).

Las relaciones entre el Estado republicano y la jerarquía católica han sido examinadas desde una perspectiva jurídica o politológica en los libros de Concha García Prous, Relaciones Iglesia-Estado en la Segunda República española (1996), Carmen de Frías, Iglesia y Constitución: la jerarquía católica ante la II República (2000) y Juan Ferreiro, Relaciones Iglesia-Estado en la II República española (2005). Muy útil es el libro colectivo editado por Julio de la Cueva y Feliciano Montero Laicismo y catolicismo: el conflicto político-religioso en la Segunda República, que aborda los proyectos y políticas secularizadoras, las reacciones y adaptaciones católicas y los principales ámbitos de confrontación, entre los que destacaban el ámbito escolar y la regulación de las órdenes 
religiosas. La Acción Católica y sus relaciones con la CEDA han sido examinadas en otro libro colectivo, coordinado también por Feliciano Montero, La Acción Católica en la II República (2008).

Eduardo González Calleja, autor de una importante producción historiográfica en los últimos años, ha publicado en 2014 En nombre de la autoridad, un estudio sobre los problemas de orden público, que contribuyeron mucho a minar la estabilidad de la democracia republicana. El episodio de violencia política más grave antes del estallido de la guerra civil fue la insurrección de octubre de 1934, sobre la que David Ruiz ha publicado una nueva síntesis general, Octubre de 1934 (2006). Sandra Souto ha estudiado sus antecedentes, desarrollo y consecuencias en el caso de Madrid en su libro ¿Y Madrid? ¿Qué hace Madrid? (2004) y Manel López Esteve ha estudiado el específico caso catalán en Els fets del 6 d'octubre de 1934 (2014).

Un tema particularmente polémico sobre el que se han publicado en los últimos años varias obras importantes es el de la relación entre el clima de enfrentamiento que se vivió en la primavera de 1936 y el subsiguiente estallido de la guerra civil. Stanley Payne lo abordó en El colapso de la República: los origenes de la guerra civil, 1933-1936 (2005), llegando a una conclusión radical: «El carácter y la extensión del colapso del orden público con el gobierno republicano de izquierda de 1936 carecen de un precedente histórico de tales proporciones en la Europa occidental». En particular destaca Payne que el gobierno no hizo un esfuerzo serio por contener la violencia de ambas partes y para mantenerse en el poder se apoyaba en los socialistas, que eran «la principal fuente de violencia». Muy distinta es la conclusión a la que llega Rafael Cruz en su libro En el nombre del pueblo: República, rebelión y guerra en la España de 1936 (2006), en el que da una cifra de víctimas mortales de la violencia política en aquellos meses muy similar al de Payne, pero observa que algo más de la mitad de ellas eran militantes de izquierda y que cerca de la mitad de quienes las causaron eran miembros de las fuerzas de seguridad o de las fuerzas armadas. La deducción obvia era que buena parte de las muertes se produjeron en enfrentamientos entre fuerzas del orden y militantes de izquierda, lo que le lleva a matizar las tesis de Payne acerca de la pasividad del gobierno frente a la violencia izquierdista y la responsabilidad de los socialistas: sólo una de cada cinco víctimas pereció a manos de la izquierda.

Uno de los epígrafes del libro de Cruz se titula «el gran miedo» y alude a que «entre distintos grupos sociales existía una sensación de peligro y amenaza». Resulta obvia, aunque no se explicite, la referencia a la Grande Peur de la Revolución Francesa, al pánico que se difundió en los campos en el verano de 1789 por el rumor de que existía una conspiración aristocrática para armar a los bandidos, lo que contribuyó a la movilización antiseñorial de los campesinos. El miedo es un sentimiento y por tanto no se explica sólo por los hechos, sino por las percepciones, fundadas o no, que se tienen. La expresión ha sido retomada por Gabriele Ranzato en La grande paura del 1936: come la Spagna precipitò nella guerra civile (2011), cuya tesis es que el temor a la revolución, que favoreció notablemente al alzamiento militar, no se circunscribía a las clases dominantes, hostiles a toda reforma que redujera su poder, sino que estaba ampliamente difundido, sobre todo entre las clases medias y los católicos de toda condición social.

Los protagonistas principales de la experiencia democrática de los años treinta fueron los partidos republicanos, cuyos líderes encabezaron casi todos los gobiernos hasta después de iniciada la guerra civil. El de mayor raigambre histórica era el Partido Radical, sobre el cual Nigel Townson publicó un libro esencial para comprender la historia política de la República, The crisis of democracy in Spain: centrist politics under the Second Republic, 
1931-1936 (2001), en el que muestra como el partido de Lerroux pudo haber sido, pero no supo ser, un factor de moderación y consolidación de la democracia republicana. Respecto a la otra corriente republicana de centro, que tuvo como principal líder al controvertido primer presidente de la República, Niceto Alcalá Zamora, hay que mencionar la obra de Luis Iñigo, La derecha liberal en la Segunda República española (2000), centrada en los partidos de esta orientación, y el estudio biográfico de Julio Gil Pecharromán: Niceto Alcalá Zamora: un liberal en la encrucijada (2005).

Sobre la izquierda republicana, Juan Avilés ha publicado La izquierda burguesa y la tragedia de la II República (2006), versión renovada de una obra anterior, que analiza la trayectoria de unos partidos que jugaron un papel decisivo en la política española tanto en el primer bienio reformista de la República como en la convulsa primavera de 1936. La figura más notable de esa corriente política fue por supuesto Manuel Azaña, una figura que sigue despertando un gran interés entre los historiadores. La principal biografía es la de Santos Juliá, Vida y tiempo de Manuel Azaña (2008), un libro que destaca por la calidad de su estilo narrativo, pero hay que mencionar también las de Ángeles Egido, Mamuel Azaña, entre el mito y la leyenda (1998) y José María Marco, Azaña (1990), varias obras colectivas, como las actas de un coloquio celebrado en Montauban en 1990, editadas por Jean-Pierre Amalric y Paul Aubert, Azaña et son temps (1993), y las recopilaciones de ensayos editada por Alicia Alted, Ángeles Egido y María Fernanda Mancebo, Manuel Azaña, pensamiento y acción (1996) y por la propia Egido, Azaña y los otros (2001). Otro estudio biográfico importante es el de Leandro Álvarez Rey: Diego Martínez Barrio: palabra de republicano (2007).

El amplio panorama de publicaciones sobre el republicanismo contrasta con la menor abundancia de los estudios sobre el socialismo. Disponemos sin embargo de un importante estudio regional, Socialismo, República y revolución en Andalucia, 1931-1936 (2000), en el José Manuel Macarro analiza las contradicciones y los enfrentamientos internos que caracterizaron la historia de las organizaciones socialistas en aquellos años. Y también se han publicado estudios interesantes sobre algunos de los principales líderes socialistas del período. Sobre Largo Caballero pueden consultarse la obra de Juan Francisco Fuentes, Largo Caballero, el Lenin español (2005) que destaca su papel en la radicalización del socialismo a partir de 1933 y la monumental del gran historiador recientemente desaparecido Julio Aróstegui, Francisco Largo Caballero: una biografia (2013), mientras que Octavio RuizManjón ha publicado una biografía de Fernando de los Ríos, un intelectual del PSOE (2007).

A diferencia del PSOE, cuya pertenencia a la Internacional Socialista tenía escasa relevancia práctica, el Partido Comunista de España de hecho formaba parte de un partido supranacional, era la sección española de la Internacional Comunista, cuyas orientaciones marcaban las líneas maestras de la política del partido. Debido a ello, la correspondencia cruzada entre la dirección del PCE y la cúpula de la Internacional en Moscú presenta un gran interés histórico, como se ha demostrado cuando, tras la desaparición de la Unión Soviética, sus archivos se han abierto a los investigadores. Fruto de ello ha sido un libro de Antonio Elorza y Marta Bizcarrondo, Queridos camaradas: la Internacional Comunista y España, 1919-1939 (1999), que representa una aportación fundamental a la historia del comunismo español en los años de la República. Por otra parte, el peso de la dirección comunista soviética sobre las decisiones de la Internacional, de donde se derivaba un complejo juego entre la política soviética, la línea de la Internacional y las decisiones del PCE, que había de aplicar esa línea en las particulares circunstancias de España. Este es el tema del interesante libro de Payne Unión Soviética, comunismo y revolución en España, 1931-1939 (2003). 
La historiografía sobre el anarquismo español durante la Segunda República ha experimentado también un avance considerable, aunque menor que en el caso del comunismo. La mejor síntesis es la de Julián Casanova, De la calle al frente: el anarcosindicalismo en España, 1931-1939 (1997), que subraya como el ciclo de insurrecciones protagonizado por la CNT en el primer bienio republicano se tradujo en una radical caída de la afiliación. Una profundización en el tema de la afiliación y del funcionamiento interno de la CNT se encuentra en la obra de Anna Monjo Militants: participació $i$ democracia a la CNT als anys trenta (2003).

Respecto a las organizaciones de derechas, el interés de los investigadores se ha centrado en los últimos años no tanto en la gran organización posibilista que era la CEDA, como en los sectores más radicales, dispuestos a acabar con la República. El sector de la derecha católica que experimentó una mayor radicalización en los años de la República, la Juventud de Acción Popular, ha sido estudiado por Sid Lowe en una obra, Catholicism, war and the foundation of Francoism (2010), que sostiene la tesis de la contribución fundamental de la CEDA a que surgiera el clima favorable en que se gestó el alzamiento militar de 1936: «la guerra -escribe Lowe- representó la continuación de la cruzada de la JAP contra la República». Una posición que contrasta con el análisis más matizado que de la actitud de la CEDA ante la democracia republicana han llevado a cabo autores como Manuel Álvarez Tardío, sobre todo en su ensayo sobre el tema en Palabras como puños, la obra colectiva dirigida por Fernando el Rey.

Acerca de la derecha alfonsina, que se alejó de los postulados liberales de la monarquía constitucional para defender una salida autoritaria para los problemas de España, destacan los libros de Julio Gil Pecharromán, Conservadores subversivos: la derecha autoritaria alfonsina, 1913-1936 (1994), y de Pedro Carlos González Cuevas, Acción Española: teologia política y nacionalismo autoritario en España, 1913-1936 (1998). La tesis de González Cuevas es que la ideología de la revista Acción Española, la más influyente en el plano intelectual, y la del conjunto de la derecha monárquica, representaban una manifestación tardía del tradicionalismo, que fundamentaba la acción política en las creencias religiosas y representaba el punto de vista de la aristocracia frente al cambio social. Hay que destacar también la documentada biografía de quien fue la gran figura de la derecha monárquica en los años de la República, escrita por Alfonso Bullón de Mendoza, José Calvo Sotelo (2004), que le presenta como «un tenaz defensor de Dios, la Patria y el Rey», aunque nunca fue tradicionalista, y partidario de «un Estado autoritario que garantizase la armonía social», que en sus últimos meses aceptó definirse como fascista, pero cuya profunda radicalización no fue un caso excepcional en su tiempo.

Una útil aproximación a la historia de la Comunión Tradicionalista en la Segunda República es la que realiza Cristina Barreiro a través del estudio de su prensa, El carlismo y su red de prensa en la Segunda República (2003), en el que destaca la contribución de los medios de comunicación tradicionalistas a la caída de la República, mediante una campaña radical de descrédito de las instituciones que planteaba el empleo de la fuerza como la única vía de salvación para España. En cuanto a la relación de los carlistas con la Iglesia española y el Vaticano, es interesante la obra de Antonio Manuel Moral Roncal, La cuestión religiosa en la Segunda República española: Iglesia y carlismo (2009).

Respecto al fascismo español, José Luis Rodríguez Jiménez, estudioso de la extrema derecha en España y en Europa, es el autor de una útil sintesis, Historia de Falange Española de las JONS (2000), que cubre desde la primera recepción de la ideología fascista italiana hasta la «domesticación» de Falange por Franco, en los primeros años de su régimen. 
Respecto al fundador de la Falange, Julio Gil Pecharromán ha escrito una buena biografía, que destaca por su ponderación: José Antonio Primo de Rivera, retrato de un visionario (1996). Finalmente, acerca del conjunto de las derechas extremas, Eduardo González Calleja ha publicado recientemente un libro en que enfatiza su creciente proclividad hacia el empleo de la violencia: Contrarrevolucionarios; radicalización violenta de las derechas durante la Segunda República, 1931-1936 (2011), en el que enfatiza como, a pesar de su diversidad, «a la altura de 1936 todas las formaciones contrarrevolucionarias estaban de acuerdo en que era la fuerza militar quien debía acabar con la República». La gran cuestión que González Calleja no aborda fue la de en como interactuó la radicalización violenta de las derechas con la de las izquierdas obreristas para generar un clima favorable a la guerra civil, una cuestión que, como hemos visto ocupa un lugar central en los diferentes análisis del colapso de la República por parte de autores como Stanley Payne, Rafael Cruz, Gabriele Ranzato o Fernando del Rey.

La selección de libros en la que se basa este comentario historiográfico deja inevitablemente fuera muchas obras de interés, pero basta para mostrar el grado en que se han renovado en las dos últimas décadas los estudios sobre el período republicano. A libros como los de Albert Balcells (Crisis económica y agitación social en Cataluña, 1931-1936, 1971), Mercedes Cabrera (La patronal ante la II República: organizaciones y estrategia, 1931-1936, 1983), Santos Juliá (Origenes de Frente Popular en España, 1979), Edward Malefakis (Reforma agraria y revolución campesina en la España del siglo XX, 1971), José Ramón Montero (La CEDA: el catolicismo social y politico en la II República, 1977), Paul Preston (La destrucción de la democracia en España: reacción, reforma y revolución en la II República, 1978), Richard Robinson (Los origenes de la España de Franco, 1974), Octavio Ruiz-Manjón (El Partido Republicano Radical, 1908-1936, 1976), Manuel Tuñón de Lara (La II República, 1976) y Javier Tusell (Historia de la democracia cristiana en España, 1974), que en los años setenta y ochenta sentaron las bases de un conocimiento sólido de la historia de la República, algunos de los cuáles siguen siendo hoy de consulta obligada, se ha añadido una nueva generación de estudios y no hay ningún síntoma de que el interés de los investigadores sobre el tema vaya a decaer en los próximos años.

El innegable avance de la investigación histórica sobre el período republicano en los últimos años se ha visto sin embargo perjudicado por un factor no menos innegable: el alto grado de politización que ha contaminado el debate historiográfico. La reivindicación de la República y la condena de la Transición se han convertido en señas de identidad de un amplio sector de las izquierdas españolas, en virtud de una opción legítima cuya funcionalidad política no tiene sentido discutir aquí. Creo sin embargo que esa actitud ha dificultado el debate razonado y documentado sobre los rasgos esenciales de aquel período histórico, que no puede consistir en estar a favor o en contra de la República, sino en establecer qué ocurrió en la España de aquellos años y en preguntarse por qué ocurrió.

El prólogo a un importante libro colectivo publicado, justo en los días en que este ensayo se concluye, por Eduardo González Calleja, Francisco Cobo Romero, Ana Martínez Rus y Francisco Sánchez Pérez (La Segunda República Española, 2015) plantea importantes cuestiones a este respecto. Su premisa es que «la vigencia de las esperanzas, los problemas y las soluciones que se suscitaron en España durante la Segunda República» se manifiesta en el hecho de que todavía no se la pueda analizar «prescindiendo de las opiniones políticas que, sobre aquel pasado, y sobre los tiempos actuales, tienen los historiadores». Yo más bien diría que algunos historiadores parecen confundir su nostalgia por una izquierda más 
agresiva con el análisis riguroso. ¿De verdad alguien cree que las soluciones propuestas hace ochenta años tienen validez en la España de hoy?

Lo dudo, aunque creo que sería interesante debatirlo en detalle, pero la tarea fundamental del historiador es otra: entender aquella España tan distinta de la nuestra. $Y$ en lo referente a las fuerzas políticas de la Segunda República creo que el diagnóstico que hace el citado prólogo puede ser compartido por cualquiera que haya estudiado seriamente el período: los grupos netamente republicanos tenían una concepción «patrimonial» de la República, a la que querían «recuperar» (Lerroux en 1932) o «no perder» (Azaña en 1934); gran parte de los socialistas y de los cedistas tenían una concepción «instrumental» de la misma, pues consideraban el régimen parlamentario como un mero punto de arranque para «una transformación radical del sistema democrático en un sentido no liberal»; y finalmente la extrema izquierda y la extrema derecha tenían una concepción «antagonista» de pleno rechazo.

De ese diagnóstico parecería razonable deducir que la composición de las fuerzas políticas no era en absoluto apropiada para la consolidación de la democracia republicana, pero me temo que González Calleja, Cobo Romero, Martínez Rus y Sánchez Pérez considerarían que esta conclusión se situa en el marco de «la historiografía de sesgo más conservador», la que caracteriza a la Segunda República como una «democracia en crisis, incompleta y deficiente». En su opinión, cualquier caracterización de la democracia española de los años treinta supone un pecado «de anacronismo y de esencialismo», porque «la democracia significa, tanto antes como ahora, muchas cosas diferentes para mucha gente». Por supuesto, pero no parece posible realizar un análisis riguroso de ningún fenómeno, sea desde la perspectiva de las ciencias sociales o de la historia, si no se emplean conceptos definidos de una manera mínimamente precisa; no se puede realizar un análisis histórico de una experiencia democrática partiendo de la base de que el término democracia puede significar cualquier cosa. De hecho, los autores parecen disponer de alguna vara de medir la autenticidad democrática, pues afirman que la Segunda República fue «el primer régimen auténticamente democrático de nuestra historia».

¿No sería pues conveniente hacer explícitos los criterios que se estiman necesarios para que un sistema se considere democrático? Existe una amplia literatura politológica al respecto, pero creo que se podrían concretar en la garantía de las libertades civiles y los derechos políticos de todos los ciudadanos y ciudadanas, en el ejercicio del poder legislativo por unas cámaras elegidas por el conjunto de la ciudadanía, en el ejercicio del poder ejecutivo por un gobierno responsable ante esas cámaras y por tanto ante la ciudadanía, y en la resolución de conflictos a través de las vías establecidas por la Constitución y las leyes. Si incluimos la exigencia de igualdad de derechos políticos para las mujeres, requisito que hoy nadie discutiría, el primer Estado soberano que gozó de una democracia plena fue Noruega, a partir de 1913, mientras que Gran Bretaña alcanzó ese estadio en 1928 y Francia en 1945 (si nos referimos a sus territorios metropolitanos, no por supuesto a sus colonias). Y desde esa perspectiva hay que destacar un hecho fundamental para nuestro tema: los mecanismos institucionales establecidos en la Constitución española de 1931 eran plenamente democráticos de acuerdo con el concepto que he expuesto y no hay pues anacronismo alguno en emplearlo para analizar la España de los años treinta. La cuestión es si en aquella España había, además de una Constitución democrática, muchos demócratas convencidos, es decir si había muchos españoles dispuestos a respetar los principios constitucionales, aunque gracias a ellos fueran sus adversarios los que gobernaran. La respuesta es que no. 


\section{Obras citadas:}

Abelló, Teresa y Solé Sabaté, J. M., eds., Lluis Companys, president de Catalunya: biografia humana i politica, Barcelona, Generalitat de Catalunya, 2007.

Alted, Alicia, Egido, Ángeles Egido y Mancebo, María Fernanda, eds., Manuel Azaña, pensamiento y acción, Madrid, Alianza Editorial, 1996.

Álvarez Rey, Leandro, Diego Martínez Barrio: palabra de republicano, Ayuntamiento de Sevilla, 2007.

Álvarez Tardío, Manuel, Anticlericalismo y libertad de conciencia, Madrid, Centro de Estudios Políticos y Constitucionales, 2002.

Álvarez Tardío, Manuel y Rey, Fernando del, eds., El laberinto republicano: La democracia española y sus enemigos, 1931-1936, Madrid, RBA Libros, 2012.

Álvarez Tardío, Manuel y Villa, Roberto, El precio de la exclusión: la politica durante la Segunda República, Madrid, Encuentro, 2010.

Amalric, Jean-Pierre et Aubert, Paul, eds., Azaña et son temps, Madrid, Casa de Velázquez, 1993.

Aróstegui, Julio, Francisco Largo Caballero : una biografia, Madrid, Debate, 2013.

Avilés, Juan, La izquierda burguesa y la tragedia de la II República, Comunidad de Madrid, 2006.

Ballarín, M. y Ledesma, J.L., eds., Avenida de la República, Zaragoza, Cortes de Aragón, 2007.

Ballarín, M., CuCAlón, D. y Ledesma, J.L., eds., La II Republica en la encrucijada: el segundo bienio, Zaragoza, Cortes de Aragón, 2009.

Ballarín, M. y Ledesma, J.L., eds., La República del Frente Popular: reformas, conflictos y conspiraciones, Zaragoza, Fundación Rey del Corral de Investigaciones Marxistas, 2010.

Barreiro Gordillo, Cristina, El carlismo y su red de prensa en la Segunda República, Madrid, Actas, 2003.

Barrios RozÚA, Juan Manuel, Iconoclastia, 1930-1936: la ciudad de Dios frente a la modernidad, Universidad de Granada, 2007.

Beramendi, Justo G. y Maiz, Ramón, Los nacionalismos en la España de la II República, Madrid, Siglo XXI, 1991.

Casanova, Julián, De la calle al frente: el anarcosindicalismo en España, 1931-1939, Barcelona, Crítica, 1997.

Casanova, Julián, República y guerra civil, Historia de España, vol. 8, Madrid, Crítica y Marcial Pons, 2007.

Chaput, Marie-Claude et Gomez, Thomas, Histoire et mémoire de la Seconde République espagnole, Paris, Université Paris X - Nanterre, 2002.

Cruz, Rafael, En el nombre del pueblo: República, rebelión y guerra en la España de 1936, Madrid, Siglo XXI, 2006.

Cueva, Julio de la y Montero, Feliciano, Laicismo y catolicismo: el conflicto político-religioso en la Segunda República, Universidad de Alcalá, 2009.

Culla i Clarä, Joan B., Esquerra Republicana de Catalunya 1931-2012: una història politica, Barcelona, La Campana, 201

Di FeBo, Giuliana e Natoli, Claudio, Spagna anni Trenta: società, cultura, istituzioni, Milano, Franco Angeli, 1993.

EGido, Ángeles, Manuel Azaña, entre el mito y la leyenda, Valladolid, Junta de Castilla y León, 1998.

EGido, Ángeles, ed., Azaña y los otros, Madrid, Biblioteca Nueva, 2001.

EgIDo, Ángeles, ed., Memoria de la Segunda República: mito y realidad, Madrid, Biblioteca Nueva y CIERE, 2006.

Elorza, Antonio y Bizcarrondo, Marta, Queridos camaradas: la Intemacional Comunista y España, 1919-1939, Barcelona, Planeta, 1999. 
Ferreiro Galguera, Juan, Relaciones Iglesia-Estado en la II República española, Barcelona, Atelier, 2005.

Frias Garcia, Carmen de, Iglesia y Constitución: la jerarquía católica ante la II República, Madrid, Centro de Estudios Políticos y Constitucionales, 2000.

Fuentes, Juan Francisco, Largo Caballero, el Lenin español, Madrid, Síntesis, 2005.

Garcia Prous, Concha, Relaciones Iglesia Estado en la Segunda República española, Córdoba, Cajasur, 1996.

Gil Pecharromán, Julio, Conservadores subversivos: la derecha autoritaria Alfonsina, 19131936, Madrid, Eudema, 1994.

Gil Pecharromán, Julio, José Antonio Primo de Rivera: retrato de un visionario, Madrid, Temas de Hoy, 1996.

Gil Pecharromán, Julio, Historia de la Segunda República española, 1931-1936, Madrid, Biblioteca Nueva, 2002.

Gil Pecharromán, Julio, Niceto Alcalá Zamora: un liberal en la encrucijada, Madrid, Síntesis, 2005.

GonzÁlez Calleja, Eduardo, Contrarrevolucionarios; radicalización violenta de las derechas durante la Segunda República, 1931-1936, Madrid, Alianza Editorial, 2011.

GonzÁlez CALLEJA, Eduardo, En nombre de la autoridad: la defensa del orden público durante la Segunda República española (1931-1936), Granada, Comares, 2014.

González Calleja, E. y Navarro, R., La España del Frente Popular: politica, sociedad, conflicto y cultura en la España de 1936, Granada, Comares, 2012.

González Calleja, E., Cobo Romero, F., Martínez Rus, A. y Sánchez Pérez, F., La Segunda República, Barcelona, Pasado y Presente, 2015.

González Cuevas, Pedro Carlos, Acción Española: teología politica y nacionalismo autoritario en España, 1913-1936, Madrid, Tecnos, 1998.

Granta, José Luis de la, El oasis vasco: el nacimiento de Euskadi en la República y la Guerra Civil, Madrid, Tecnos, 2007.

Ínigo FeRnÁndez, Luis, La derecha liberal en la Segunda República española, Madrid, UNED, 2000.

Juliá, Santos, ed., República y guerra en España, Madrid, Espasa, 2006.

Juliá, Santos, Vida y tiempo de Manuel Azaña: Madrid, Taurus, 2008.

López Esteve, Manel, Els fets del 6 d'octubre de 1934, Barcelona, Base, 2014.

LóPEZ VILLAVERDE, Ángel Luis, El gorro frigio y la mitra frente a frente: construcción y diversidad territorial del conflicto político-religioso en la España republicana, Barcelona, Rubeo, 2008.

Lowe, Sid, Catholicism, war and the foundation of Francoism: the Juventud de Acción Popular in Spain, 1931-1939, Brighton, Sussex Academic Press, 2010.

Macarro Vera, José Manuel, Socialismo, República y revolución en Andalucia, 1931-1936, Universidad de Sevilla, 2000.

Marco, José María, Azaña, Madrid, Mondadori, 1990.

Mees, L., Granja, J.L. de la, Pablo, S. de y Rodríguez Ranz, J.A., La politica como pasión: el lehendakari José Antonio Aguirre (1904-1960), Madrid, Tecnos, 2014.

Monso, Anna, Militants: participació i democracia a la CNT als anys trenta, Barcelona, Laertes, 2003.

Montero, Feliciano, ed., La Acción Católica en la II República, Universidad de Alcalá, 2008.

Moral Roncal, Antonio Manuel, La cuestión religiosa en la Segunda República española: Iglesia y carlismo, Madrid, Biblioteca Nueva, 2009.

Payne, Stanley, Spain's first democracy: the Second Republic, 1931-1936, Madison, The University of Wisconsin Press, 1993. (Traducción española: La primera democracia española, Barcelona, Paidós, 1995). 
PAyne, Stanley, Unión Soviética, comunismo y revolución en España, Barcelona, Plaza Janés, 2003. (Edición inglesa: The Spanish Civil War, the Soviet Union, and communism, New Haven, Yale University Press, 2004).

PAYne, Stanley, El colapso de la República: los origenes de la guerra civil, 1933-1936, Madrid, La Esfera de los Libros, 2005. (Edición inglesa: The collapse of the Spanish Republic, New Haven, Yale University Press, 2006).

RANZATo, Gabriele, L'eclissi della democracia: la guerra civile spagnola e le sue origini, 19311939, Torino, Bolati Boringhieri, 2004. (Traducción española: El eclipse de la democracia, Madrid, Siglo XXI, 2006).

RanZato, Gabriele, La grande paura del 1936: come la Spagna precipitò nella guerra civile, Bari, Laterza, 2011. (Traducción española: El gran miedo de 1936, Madrid, La Esfera de los Libros, 2014).

Rey, Fernando del, ed., Palabras como puños: la intransigencia politica en la Segunda República española, Madrid, Tecnos, 2011.

Rodriguez Jiménez, José Luis, Historia de Falange Española de las JONS, Madrid, Alianza Editorial, 2000.

Ruiz, David, Octubre de 1934: revolución en la República española, Madrid, Síntesis, 2006.

Ruiz-Manjón, Octavio, Fernando de los Ríos, un intelectual del PSOE, Madrid, Síntesis, 2007.

Souto Kustrin, Sandra: ¿Y Madrid? ¿Qué hace Madrid? Movimiento revolucionario y acción colectiva (1933-1936), Madrid, Siglo XXI, 2004.

Тномаs, Maria: La fe y la furia: violencia anticlerical popular e iconoclastia en España, 19311936, Granada, Comares, 2014.

Townson, Nigel, The crisis of democracy in Spain: centrist politics under the Second Republic, 1931-1936, Eastbourne, Sussex Academic Press, 2001. (Traducción española: La República que no pudo ser, Madrid, Taurus, 2002). 